\title{
Horizons/Théâtre
}

Revue d'études théâtrales

8-9 | 2016

Théâtres du geste, du jeu et de la voix

\section{Survivances carnavalesques au Maroc}

\section{Mouna Hachim}

\section{(2) OpenEdition}

Journals

Édition électronique

URL : https://journals.openedition.org/ht/852

DOI : $10.4000 /$ ht. 852

ISSN : 2678-5420

\section{Éditeur}

Presses universitaires de Bordeaux

\section{Édition imprimée}

Date de publication : 31 décembre 2016

Pagination : 162-170

ISSN : 2261-4591

\section{Référence électronique}

Mouna Hachim, "Survivances carnavalesques au Maroc ». Horizons/Théâtre [En ligne], 8-9 | 2016, mis en ligne le 01 janvier 2018, consulté le 17 mai 2022. URL : http://journals.openedition.org/ht/852 DOI : https://doi.org/10.4000/ht.852

\section{(c) $(1) \odot$}

La revue Horizons/Théâtre est mise à disposition selon les termes de la Licence Creative Commons Attribution - Pas d'Utilisation Commerciale - Pas de Modification 4.0 International. 


\section{MOUNA HACHIM}

Mouna Hachim est une chercheuse et une femme de lettres marocaine. Romancière, chroniqueuse, ses recherches sont axées sur une double culture et un travail de translation de l'arabe vers le français. Mouna Hachim écrit ainsi en français à partir de recherches historiques anciennes et contemporaines qui sont pour l'essentiel en langue arabe. Elle est l'auteur d'un roman, Les Enfants de la Chaovia (Casablanca, Autoédition, 2004), du Dictionnaire des noms de famille du Maroc (Casablanca, Autoédition, 2007 - Nouvelle édition augmentée, Casablanca, Édition Le Fennec, 2011) et de Chroniques insolites de notre histoire (Maroc, des origines à 1907) (Casablanca, Autoédition, 2016). Elle est également l'auteur d'une série documentaire pour la chaîne TV Medi 1 sur la « route des origines » autour de ses recherches sur la généalogie et les noms de famille du Maroc.

Mail : mounahachim1@gmail.com

Résumé: Chaque année, se déroulent au Maroc des festivités qui sont l'occasion d'observer des réminiscences vivaces, sous une forme plus ou moins édulcorée, de rites carnavalesques, caractérisés par des spectacles burlesques et des processions masquées. C'est le cas de la cérémonie de Boujoud (l'Homme aux peaux), survenant juste après la grande fête du Sacrifice. C'est le cas également de Achoura, représentation de l'année qui meurt et ses symbolismes liés à l'organisation du monde et à la permanence cyclique de la vie et de la mort. Que dire de Yennayer dont les symbolismes universels de « portes de l'année » étaient l'occasion

Abstract: There are festivities every year in Morocco which are times of lively reminiscences - in more or less watered-down forms -- of carnival rituals characterized by burlesque shows and by masked processions. It is the case of the Boujloud ceremony (The Man with Skins) which takes place just after the great Sacrifice festivity. It is also the case of Achoura, a representation of the waning year along with its symbolism in connection with the organization of the world and with the cyclical permanence of life and death. What then of Yennayer whose universal symbolisms as "doors of the year" were the time of great festivities throughout the dans tout le Maghreb de grandes festivités dont le point commun étaient des rites de purification, de renouvellement et de placement de la nouvelle année sous les meilleurs augures? Sans oublier le surprenant Sultan des étudiants qui voyait l'intronisation par les étudiants des medersas d'un roi-bouffon. Autant d'expressions originales du vécu, du légué, du pensé, du ressenti, du créé... qui étonnent par leur permanence malgré la captation par le monothéisme et la méfiance des pouvoirs.

Mots-clés : spectacle, carnaval, Boujloud, Maroc

Maghreb region and whose common point were the rituels of purification, of renewal and of placing the new year under better omens? Nor should we left aside the remarkable Sultan of students which marked the enthronement of by the students of medersas of a buffoon-king. These are all original manifestations of real life experiences, of heritage, of thoughts, of perception, of creation, etc., which impress by their perenial nature in spite of recuperation by monotheism and the mistrust of the State.

Keywords: show, Morocco, Boujloud, carnival

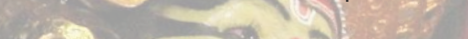




\section{Survivances carnavalesques au Maroc}

Chaque année, juste après la célébration de la grande fête du Sacrifice se déroulait à travers les villes et campagnes du Maroc, avec des rémanences encore vivaces, sous une forme plus ou moins édulcorée, dans les montagnes du Haut-Atlas et de l'Anti-Atlas ainsi que dans les vallées du Souss, une surprenante fête populaire, caractérisée par ses spectacles burlesques et ses processions masquées.

Appelée Herma (de la racine HRM évoquant la vieillesse) ou Chouiekh (littéralement, le petit vieux), elle est dite aussi selon les régions et les parlers berbérophones ou arabophones, Bilmawen, Bou-Islikhnen, Bou-l-Btayn, Bou-Hidour... ou Bou-Jloud, soit l'Homme aux peaux.

Le lendemain du jour du sacrifice rituel, le personnage principal de la mascarade émanait au milieu de chaque groupement, accoutré de peaux fraîches de moutons ou de chèvres munies de leurs sabots pendouillant au bout des bras, paré d'un collier de coquilles d'escargots faisant parfois usage de chapelet, le visage peinturluré en noir, affublé d'un masque hideux représenté parfois par une vieille outre, la tête ornée de cornes ou de tête de mouton à la mâchoire écartée, grimaçante, tout en veillant à mettre en valeur de puissants attributs sexuels, exhibés sous forme d'organes de reproduction de la bête ou d'un quelconque symbole de phallus géant assorti de deux aubergines.

C'est avec ce déguisement grotesque, connaissant des variantes selon les régions, que s'effectuait de hameau en hameau ou d'une rue à l'autre en milieu citadin, la tournée de Boujloud, au rythme des musiques des hautbois et des tambourins et d'exubérants spectacles dignes d'un authentique carnaval.

Comme dans un geste de conjuration des influences néfastes, Boujloud avait pour mission de toucher, voire de frapper à l'aide de son long bâton ou de pattes de bouc ou de mouton, les grands et les petits, ainsi que les piliers des maisons ou des tentes visitées.

Cette ambiance singulière durait plusieurs jours, marquée par des spectacles de rue, animée par des acteurs qui effectuaient leur ronde réjouissante auprès de Boujloud, soldée par des oboles sous forme d'œufs, de sucre, de 
blé ou de viande et se prolongeant jusqu'à des heures tardives sur la place publique. C'est ce qui explique le nom laissé à Bab-Boujloud, un des principaux accès de la médina de Fès ou à l'esplanade de la fameuse porte Boujloud à Marrakech.

Parmi les autres personnages stéréotypés de ces mascarades figurent le vieillard (appelé Ba-Cheikh), le juif et sa femme, le cadi aux jugements extravagants, l'âne, le voleur, l'officier français, l'agent d'autorité marocain (mokhazni), l'ambassadeur...

Ils offraient tous leurs Fraja, spectacles et autres représentations, ouvertes sur les places publiques, exposées devant les notabilités et les sultans, parodiant la justice, la politique ou la religion avec une liberté de ton et un sens de la dérision exceptionnels, autorisés en ce temps et espace de remise en cause. C'est d'ailleurs la raison qui expliquerait l'interdiction de ces réunions bigarrées par les autorités coloniales lesquelles, tout en tolérant leur fonction d'exutoire, ne pouvaient ignorer les dangers de leur regard critique sur l'organisation sociale et politique et leur inversion des normes établies, avec les risques de contestation et de débordement qui en découlent.

De même, l'Indépendance amènera des soubresauts réformistes aussi élitistes que conservateurs qui ont tenté d'interdire l'expression de ces représentations populaires au nom de la morale, condamnant ces vestiges primitifs, cette rupture des inhibitions et cette régression vers une forme animale.

Il faut dire que les études de ces traditions anciennes en Afrique du Nord ont passionné les voyageurs et ethnologues étrangers qui les ont décortiquées depuis le début du $\mathrm{xx}^{\mathrm{e}}$ siècle, essentiellement sous le prisme des survivances du paganisme dans la civilisation musulmane.

Les orientalistes français Edmond Doutté ou Émile Laoust voient ainsi dans le cérémonial de Boujloud, les débris de rites magiques berbères, symbolisant l'alternance des saisons, la mort et la résurrection du dieu de la végétation.

Le Berbère n'aurait-il pas établi, jadis, aux temps déjà éloignés de son islamisation, un rapport si étroit entre le sacrifice du mouton, ordonné par l'Islam, et la procession carnavalesque d'un personnage vêtu de peaux qu'il aurait vu, en ces deux rites, deux épisodes d'une même cérémonie, s'interroge le linguiste et ethnographe Émile Laoust. [... ] nous serons amenés à conjecturer que l'Aïd el Kebir s'est substituée, en Berbérie, à une fête similaire qui existait déjà et au cours de laquelle les indigènes sacrifiaient un bélier et se revêtaient de sa dépouille. Si l'on se rappelle que le bélier fut autrefois l'objet d'un culte dont le souvenir s'est conservé tard dans le pays, on voudra peut-être voir, dans les mascarades actuel- 
lement célébrées à l'Aï el Kebir, la survivance de pratiques zoolâtriques dont l'origine se perd dans les âges obscurs de la préhistoire ${ }^{1}$.

Pour sa part, l'anthropologue finnois, Edvard Westermarck explique ces rites en remontant aux fêtes des Saturnales romaines. Le voyageur français Auguste Mouliéras décrit à ce propos au début du $\mathrm{Xx}^{\mathrm{e}}$ siècle le carnaval en pays Jbala, dans le Rif Occidental, connu sous le nom de Ba-Chikh en insistant sur son caractère débridé et licencieux apparenté aux fêtes des Saturnales, décrivant $\mathrm{Ba}$-Cheikh comme un « vieux paillard » obsédé par l'accouplement ${ }^{2}$.

Rappelons à ce propos que le culte de Saturne l'Africain est la renaissance sous une forme romanisée d'un vieux rite dédié au dieu mâle suprême Baal-Hamon qui est une divinité libyco-punique, associant le phénicien Baal à l'Africain Ammon, divinité tutélaire de Thèbes, représentée par un bélier. Commun aux Égyptiens, aux Éthiopiens et aux Libyens (ancêtres des Berbères), Ammon est honoré par les Grecs qui l'associent à Zeus et par les Romains qui le nomment Jupiter-Ammon. Il est également unifié à la divinité phénicienne Baal-Hammon, surnommée Saturne l'Africain.

Certains auteurs n'ont pas manqué de faire des analogies entre ce culte et les représentations plus anciennes du bélier avec sa coiffure de forme sphérique dans l'art rupestre préhistorique berbère même si cela ne fait pas l'unanimité, Gabriel Camps voyant dans ces gravures de l'art néolithique de l'Atlas davantage des animaux parés pour le sacrifice qu'un culte du bélier³. Un culte toutefois attesté en Afrique du Nord depuis le Néolithique et sa large diffusion confirmée par l'étendue géographique des gravures rupestres.

Quant à l'Ammon de Siouah, précise Gabriel Camps, quelles que soient ses origines exactes, il faut reconnaître que son oracle eut une réputation internationale qui dépassa largement le cadre géographique libyen. Par l'intermédiaire des Grecs de Cyrénaïque son nom, sa réputation, son effigie, complètement humanisée sous l'influence hellénique, gagnèrent le monde méditerranéen. Ammon, bientôt appelé Zeus-Ammon, est représenté sous les traits d'un personnage barbu débonnaire, ne conservant du bélier thébain que les cornes plus ou moins dissimulées dans la chevelure bouclée 4 .

Associé aux rites de fécondité, Saturne l'Africain continuait pour sa part à occuper un rang central dans le panthéon berbéro-carthaginois, avant d'être adopté par les Romains qui voient en lui le héros civilisateur enseignant la culture de la terre aux hommes.

Les Saturnales et Lupercales étaient ainsi des fêtes de la fertilité de la terre et des êtres vivants, caractérisées par la réunion de luperques, vêtus de peaux, de bouc ou de chèvres sacrifiés avant la fête, courant autour de la colline du 
Palatin et fouettant de leurs lanières les passants, particulièrement les femmes, faisant dire à Cicéron : «La sodalité sauvage, toute pastorale et agreste des frères luperques, dont le rassemblement sylvestre a été institué avant la civilisation humaine et les lois. ${ }^{5} \gg$

Tout en notant la transgression totale et paradoxale des normes instaurées pendant cette fête rituelle, l'anthropologue marocain, Abdallah Hammoudi précise dans son ouvrage La Victime et ses masques. Essai sur le sacrifice et la mascarade au Maghreb :

Une telle contradiction cadre si peu avec les théories classiques du rituel qu'on a préféré y voir un effet résiduel, où sous le rite musulman se cacheraient les survivances de coutumes romaines, chrétiennes, voire d'une religion berbère originelle. Mais une description systématique de ce drame cyclique montre qu'il n'en est rien et que sacrifice et mascarade procèdent d'un même processus rituel cohérent et orienté à travers lequel une culture se donne une représentation d'elle-même et met en scène ses propres tensions ${ }^{6}$.

Dans son intérêt pour les concepts psychanalytiques, l'auteur avance des analogies avec le rêve : «le drame décrit se présente comme un bric-à-brac, un montage indémêlable d'actions-propositions relevant du bons sens ordinaire et d'autres à première vue absurdes. »

Symboles névrotiques, survivances de rites antiques, illustration de drames cosmiques, le moins que l'on puisse dire donc, c'est que profane et sacré se mêlent en ce mois de Doul-Hijja, douzième du calendrier musulman dont les premiers jours sont dédiés au pèlerinage ; et, le dixième, marqué par la fête du sacrifice, commémorant le geste d'Abraham. Ces processions carnavalesques débutaient toutefois, selon les régions, immédiatement après la cérémonie du sacrifice ou le soir même de l'Achoura, si ce n'est pendant les deux fêtes à la fois.

\section{Achoura, mort et renouveau}

Tirant son nom de Achara (dix), Achoura est le dixième jour du mois sacré de Mouharram qui célèbre l'an I de l'Hégire, défini par la Migration du Prophète à Médine où prospéra le noyau de la première communauté musulmane.

Selon la tradition, le Prophète commença à jeûner ce jour de l'Achoura, lorsqu'à son installation à Médine, il rencontra des tribus juives en plein jeûne du Kippour, marquant la sortie d'Egypte. Le Prophète adopta alors ce jeûne et le recommanda en signe d'inscription de l'islam dans la tradition mosaïque. 
Chez les chiites, l'Achoura revêt une dimension centrale, imprégnée de mortifications, évoquant la mort en martyr de Houssein, petit-fils du Prophète, érigé au rang d'imam, assassiné à Kerbela. Cet épisode dramatique a donné naissance à un genre théâtral particulier en Iran, appelé Tazieh (taâziya, consolation) jouant les passions, portant sur les souffrances et la douleur. Un jour de deuil, hautement réprouvé par les autorités sunnites qui arguent que même les prophètes n'ont pas droit à un jour de deuil.

Différentes légendes sont rattachées à l'Achoura, aussi fantaisistes et invérifiables l'une que l'autre, évoquant la date de la repentance d'Adam ou l'accostage de l'arche de Noé, l'évacuation de Joseph du puits ou la naissance de Jésus... Mais beaucoup s'accordent à la rattacher à de vieux rites agraires, captés par les religions monothéistes successives.

Les chercheurs occidentaux ont comparé en effet ces représentations populaires en Afrique du Nord au carnaval européen, avec ses revirements de situations et ses symbolismes de mort et de renouveau, conformément à la définition du théoricien russe de la littérature, Mikhail Bakhtine.

Car si Achoura est marqué sur le plan social par ses réunions familiales conventionnelles, ses savoureux mets, ses gourmandises évocatrices de fécondité, ses jouets bruyants, ses aumônes, ses visites aux tombes..., elle reste indissociable de rituels caractéristiques de cette ambivalence portant sur la mort et sur le renouvellement de la nature avec comme représentations symboliques : les rites associant l'eau et le feu.

Avant l'aspersion des passants, le matin de l'Achoura, avec de l'eau (identifiée à l'eau bénie Zem-Zem provenant du puits mecquois) avait eu lieu dans la soirée, les feux de joie. Ce sont les fameuses Châ̂la, rapprochées des feux de la Saint-Jean se déroulant en Europe pendant le solstice d'été et vestiges eux-mêmes d'anciens cultes agraires celtes et germaniques préchrétiens.

Liées au sacré, les représentations dramatiques atteignaient leur paroxysme pendant l'Achoura comme cela a été décrit notamment par le sociologue Edmond Doutté à Marrakech ou à Fès en 1907 dans son ouvrage Magie et religion dans l'Afrique du Nord. Il consacre un chapitre entier au « Carnaval du Maghrib » avec sa floraison de personnages masqués, notamment le Petit vieillard affublé d'une citrouille sur la tête et d'une peau de hérisson en guise de barbe, ses bouffonneries, ses scènes tragiques, ses symbolismes liés à l'organisation du monde et à la permanence cyclique de la vie et de la mort...

Illustration avec cet acte significatif d'enterrement de la poupée Baba Achour ou Tisli n'Achour (la fiancée de Achour), faite généralement d'os et de chiffons, avec des danses autour de grands feux et un simulacre d'enterrement 
accompagné de pleurs, représentant la douleur de la séparation de l'année écoulée et la joie des retrouvailles d'une année nouvelle.

Cette poupée ou mannequin est par ailleurs comparée au bonhomme carnaval européen remarquable pendant le Mardi-Gras ou le Mercredi des Cendres où le personnage finissait noyé, enterré ou brûlé dans une cérémonie concluant au « parallélisme parfait des fêtes africaines et européennes $»^{7}$.

Ces fêtes symboliseraient l'année qui meurt ou représenteraient un esprit de la végétation dont le meurtre rituel laisserait transparaître ses « derniers débris $\gg$. Mais

Lorsque Achoura devient une fête du calendrier lunaire, écrit Edmond Doutté, elle ne concorde plus avec les époques agricoles; elle faisait le tour du calendrier solaire. Soit qu'elle eût jadis, réellement été une fête de renouveau, soit que son manque de consistance et son indétermination dans l'orthodoxie musulmane eussent contribué à la faire captation, des rites de l'année solaire qui ne coïncident primitivement avec elle qu'à des intervalles éloignés. Il était naturel d'ailleurs pour des populations qui s'islamisaient, de rattacher au début de l'année musulmane lunaire des cérémonies célébrées depuis un temps immémorial au début de l'année solaire ${ }^{8}$.

\section{Yennayer, « La porte de l'année »}

Aux côtés du calendrier lunaire basé sur l'observation des phases biologiques de la lune, un autre calendrier, plus ancien, scandé par le rythme des saisons était imposé par les exigences de l'agriculture, d'où son appellation de Filahi, agricole. C'est le calendrier authentique de l'Afrique du Nord, dérivé du calendrier julien qui est institué dans tout l'Empire romain par Jules César en 46 av. J.-C., sous les conseils de son astronome égyptien Sosigène d'Alexandrie.

En Afrique du Nord, le calendrier agraire, marqué par le rythme des saisons et des activités agricoles, fête les labours, les semailles et les moissons et évoque cette harmonie entre la vie humaine et la vie végétale, toutes deux soumises aux mêmes lois et aux mêmes vicissitudes.

Le premier jour de l'année de ce calendrier, correspondant au 13 janvier du calendrier grégorien est dit Yennayer, du latin Ianuarius. Ses symbolismes universels de portes de l'année étaient l'occasion dans tout le Maghreb de grandes festivités que ce soit en zone berbère ou arabe. Si les changements de traditions étaient de mise d'une région à une autre, le point commun entre toutes ces réunions festives qui duraient plusieurs jours étaient des rites de purification, de renouvellement (d'ustensiles, de pierres des foyers...) et de 
placement de la nouvelle année sous les meilleurs augures liés à l’abondance, à travers de copieux repas.

Les chants (comme l'Ahidous), les jeux et les combats rituels n'étaient pas en reste, de même que les bouffonneries et les masques autorisant la dérision, la permissivité ou représentant les mystères de l'Invisible.

\section{Le Surprenant « Sultan des Tolba »}

Comment conclure ce petit tour d'horizons de fêtes carnavalesques sans un regard sur cette autre cérémonie burlesque appelée Soltane Tolba (roi des étudiants) qui voyait l'intronisation par les étudiants des medersas d'un sultan éphémère.

Aussitôt proclamé, le roi de carnaval s'entourait de son appareil administratif de rigueur (vizirs, chambellan...), aidé en cela par le pouvoir central qui lui envoyait tenues d'apparat, montures, parasol, escorte... Entouré de sa cour, notre sultan fantoche, se mettait ensuite à parodier la gestion du pouvoir, levait des impôts, recevait des délégations et des cadeaux farfelus, avant la visite du véritable Sultan qui répondait à sa faveur. Le roi-bouffon n'avait plus alors qu'à regagner sa Médersa, échappant aux boutades de ses anciens sujets.

D'aucuns expliquent les origines de cette fête comme un geste de remerciements du premier sultan alaouite Moulay Rachid au XVII ${ }^{\mathrm{e}}$ siècle à ses anciens camarades de l'université Qaraouiyine pour leur rôle dans la lutte contre le puissant négociant juif Aaron ben Mech'al, tandis que d'autres chercheurs y voient le débris d'une antique fête printanière et le vestige de rites carnavalesques assimilés par le pouvoir.

Son influence sur le théâtre marocain contemporain est en tous les cas notable avec notamment la pièce Soltane tolba présentée dans les années 60 par le dramaturge Abdessamad Kenfaoui et interprétée par la troupe de Tayeb Saddiki.

Une étude plus poussée de ces célébrations reste toutefois à accomplir à plusieurs niveaux en tant qu'expressions originales du vécu, du légué, du pensé, du ressenti, du créé... Car si ces fêtes étonnent par la permanence maintenue au fil des siècles malgré la captation par le monothéisme et la méfiance des pouvoirs, elles risquent avec l'urbanisation, l'éloignement du travail de la terre et l'observation de la nature qui en découle, de se transformer, en contes finissants, privés de leur contexte social et mental, bousculés par de nouveaux rythmes et de nouvelles mythologies. 


\section{Notes}

1. Émile Laoust, «Noms et cérémonies des feux de joie chez les Berbères du Haut et de l'Anti-Atlas ", Hespéris, Archives berbères et Bulletin de l'Institut des Hautes Études Marocaines, Paris, Émile Larose, 1921, vol. I, p. 258.

2. Auguste Mouliéras, "Exploration des Djebala (Maroc Septentrional) ", in id., Le Maroc inconnu : étude géographique et sociologique, t. II, Impr. Fouque \& cie, 1899, p. 611.

3. Gabriel Camps, "Bélier à sphéroïde (gravure rupestre de l'Afrique du Nord) ", in Encyclopédie berbère, 9 | Baal-Ben Yasla, Aix-en-Provence, Edisud, 1991, p. 1417-1433.

4. Gabriel Camps, "Ammon ", in Encyclopédie berbère, $4 \mid$ Alger - Amzwar, Aix-enProvence, Edisud, 1986, p. 596-599.

5. Cité dans Marguerite Garrido-Hory et Antonio Gonzalès (éd.), Histoire, espaces et marges de l'Antiquité : hommages à Monique Clavel-Lévêque, vol. 3, Besançon, Presses Universitaires de Franche-Comté, série « Histoire et Politique », 2004, p. 265.

6. Abdallah Hammoudi, « La victime et ses masques. Essai sur le sacrifice et la mascarade au Maghreb », Paris, Éditions du Seuil, 1988, p. 246.

7. Emile Laoust, op. cit.

8. Edmond Doutté, Magie et religion dans l'Afrique du Nord, Alger, A. Jourdan, 1909, p. 528. 